\title{
Eine besondere Familie von Polynomen
}

\author{
Werner Hertzog \\ Werner Hertzog hat an der Universität Freiburg im Üechtland Mathematik studiert. \\ Heute ist er als Direktor der Pensionskasse des Bundes PUBLICA (Schweiz) tätig.
}

In der Formel für den Tangens eines $n$-fachen Winkels treten Polynome $p_{n}$ und $q_{n}$ in $\mathbb{Z}[x]$ auf. Diese Polynomfamilien $\left(p_{n}\right)_{n \geq 1}$ und $\left(q_{n}\right)_{n \geq 1}$ haben unerwartete Eigenschaften, welche sich mit elementaren Mitteln herleiten lassen. Beispielsweise lässt sich mit ihrer Hilfe die komplexe Wurzelfunktion auf einem maximalen Holomorphiegebiet gebrochen rational approximieren. Es handelt sich dabei um ein konkretes Beispiel für den allgemeinen funktionentheoretischen Satz von Runge [1].

Es bezeichne $G:=\mathbb{C} \backslash\{z \in \mathbb{R} \mid z \leq 0\}$ die eingeschnittene komplexe Ebene und $H:=$ $\{z \in \mathbb{C} \mid \operatorname{Re} z>0\}$ die rechte Halbebene. Wir definieren die komplexe Wurzelfunktion $\sqrt{ }$ durch ihren Hauptwert

$$
\sqrt{ }: \quad G \rightarrow H, \quad r e^{i \phi} \mapsto \sqrt{r} e^{i \phi / 2} \quad(r>0,|\phi|<\pi)
$$

und beginnen mit den für $z \in G$ definierten Hilfsfunktionen

$$
h_{n}(z):=(1+\sqrt{z})^{n}, \quad k_{n}(z):=(1-\sqrt{z})^{n} \quad(n \geq 1) .
$$

Trennt man hier die geraden und ungeraden Anteile, so erhält man Darstellungen

$$
\left.\begin{array}{l}
h_{n}(z)=q_{n}(z)+\sqrt{z} p_{n}(z) \\
k_{n}(z)=q_{n}(z)-\sqrt{z} p_{n}(z)
\end{array}\right\} \quad(z \in G)
$$

Der funktionentheoretische Satz von Runge garantiert für jede auf einem beliebigen Gebiet holomorphe Funktion eine Approximationsfolge von rationalen Funktionen, welche auf dem jeweiligen Gebiet kompakt konvergiert. Dieser Satz ist eine wichtige Existenzaussage; wie allerdings im konkreten Fall eine solche Funktionenfolge konstruiert wird, lässt er offen. Der Autor zeigt im Folgenden, wie eine solche Folge für die komplexe Wurzelfunktion auf einem maximalen Holomorphiegebiet konkret konstruiert werden kann. Der Beweis dieses Resultats ist überraschenderweise sehr kurz und elementar. 
mit Polynomen

$$
q_{n}(z)=\frac{h_{n}(z)+k_{n}(z)}{2}, \quad p_{n}(z)=\frac{h_{n}(z)-k_{n}(z)}{2 \sqrt{z}},
$$

die sich rekursiv wie folgt berechnen lassen:

Lemma 1. Für alle $n \geq 1$ gilt

$$
\begin{array}{ll}
q_{1}(z)=1, & q_{n+1}(z)=q_{n}(z)+z p_{n}(z), \\
p_{1}(z)=1, & p_{n+1}(z)=q_{n}(z)+p_{n}(z) .
\end{array}
$$

Beweis. Man hat $q_{1}(z)=\frac{1}{2}\left(\left(h_{1}(z)+k_{1}(z)\right)=1\right.$ und weiter

$$
\begin{aligned}
q_{n+1}(z) & =\frac{h_{n+1}(z)+k_{n+1}(z)}{2}=\frac{(1+\sqrt{z}) h_{n}(z)+(1-\sqrt{z}) k_{n}(z)}{2} \\
& =\frac{(1+\sqrt{z})\left(q_{n}(z)+\sqrt{z} p_{n}(z)\right)+(1-\sqrt{z})\left(q_{n}(z)-\sqrt{z} p_{n}(z)\right)}{2} \\
& =q_{n}(z)+z p_{n}(z) .
\end{aligned}
$$

Der Beweis für die $p_{n}$ verläuft analog.

Aus

$$
p_{n}(z)=\frac{h_{n}(z)-k_{n}(z)}{2 \sqrt{z}}=\frac{(1+\sqrt{z})^{n}}{2 \sqrt{z}}\left(1-\left(\frac{1-\sqrt{z}}{1+\sqrt{z}}\right)^{n}\right) \quad(z \in G)
$$

zieht man den folgenden Schluss: Die in $G$ gelegenen Nullstellen von $p_{n}$ sind Lösungen der Gleichung

$$
\left(\frac{1-\sqrt{z}}{1+\sqrt{z}}\right)^{n}=1
$$

Aus (2) folgt aber $(1-\sqrt{z}) /(1+\sqrt{z})=e^{2 k \pi i / n}$ für ein $k \in \mathbb{Z}$ und damit weiter $z=-\tan ^{2} \frac{k \pi}{n} \notin G$. In anderen Worten: Alle Nullstellen von $p_{n}$ liegen auf der negativen reellen Achse.

Es ist nun keine Überraschung mehr, dass die Polynome $p_{n}$ und $q_{n}$ in der Formel für den Tangens des $n$-fachen Winkels auftreten:

$$
\tan (n x)=\tan (x) \frac{p_{n}\left(-\tan ^{2}(x)\right)}{q_{n}\left(-\tan ^{2}(x)\right)} \quad\left(n \geq 1, q_{n}\left(-\tan ^{2}(x)\right) \neq 0\right) .
$$

Der Beweis ergibt sich ohne Schwierigkeit mit vollständiger Induktion und Lemma 1.

Im Folgenden wollen wir zeigen, wie sich die komplexe Wurzelfunktion mit Hilfe der Polynome $p_{n}$ und $q_{n}$ gebrochen rational auf einem maximalen Holomorphiegebiet kompakt konvergent approximieren lässt. Wir haben hier ein Anwendungsbeispiel für den bekannten Approximationssatz von Runge vor uns. In seiner einfachsten Form lautet dieser Satz wie folgt (siehe z.B. [1], Kap. VII, §1, Satz 1.1): 
Satz von Runge. Es sei $f$ eine auf der kompakten Menge $K \subset \mathbb{C}$ holomorphe Funktion. Dann gibt es eine Folge $\left(f_{n}\right)_{n \geq 1}$ rationaler Funktionen ohne Pole auf $K$, die gleichmässig auf $K$ gegen $f$ konvergiert.

Der Satz von Runge ist eine reine Existenzaussage. Wie die Approximationsfolge im konkreten Fall aussieht, lässt er offen.

Die Funktionen

$$
f_{n}(z):=\frac{q_{n}(z)}{p_{n}(z)} \quad(n \geq 1)
$$

sind gebrochen rational und alle in dem Gebiet $G$ holomorph, da die Nullstellen der $p_{n}$ auf der negativen reellen Achse liegen. Wir setzen

$$
\omega=\omega(z):=\frac{1-\sqrt{z}}{1+\sqrt{z}} \quad(z \in G) .
$$

Dann folgt aus (1) die Darstellung

$$
f_{n}(z)=\frac{q_{n}(z)}{p_{n}(z)}=\sqrt{z} \frac{1+\omega^{n}}{1-\omega^{n}} \quad(n \geq 1, z \in G) .
$$

Wie verhält sich $\frac{1+\omega^{n}}{1-\omega^{n}}$, wenn $n \rightarrow \infty$ ? Für festes $z=r e^{i \phi}$ mit $r>0$ und $|\phi|<\pi$ ergibt sich

$$
|\omega|^{2}=\omega \cdot \bar{\omega}=\frac{1+r-2 \sqrt{r} \cos (\phi / 2)}{1+r+2 \sqrt{r} \cos (\phi / 2)}<1 .
$$

Somit ist auch $|\omega|<1$, und es folgt $\lim _{n \rightarrow \infty} \frac{1+\omega^{n}}{1-\omega^{n}}=1$. Wegen (3) haben wir damit

$$
\lim _{n \rightarrow \infty} f_{n}(z)=\lim _{n \rightarrow \infty} \frac{q_{n}(z)}{p_{n}(z)}=\sqrt{z} \quad(z \in G)
$$

bewiesen. Wir können damit unser Hauptresultat formulieren:

Satz 2. Auf dem Gebiet $G:=\mathbb{C} \backslash\{z \in \mathbb{R} \mid z \leq 0\}$ gilt

$$
\lim _{n \rightarrow \infty} \frac{q_{n}(z)}{p_{n}(z)}=\sqrt{z}
$$

mit kompakter Konvergenz.

Beweis. Die punktweise Konvergenz ist bereits bewiesen. Es sei nun ein beliebiges Kompaktum $K \subset G$ gegeben. Wir müssen zeigen, dass die $q_{n} / p_{n}$ auf $K$ gleichmässig gegen $\sqrt{ }$ konvergieren. Das heisst: Zu jedem $\epsilon>0$ gibt es ein $N$ mit

$$
\left|\frac{q_{n}(z)}{p_{n}(z)}-\sqrt{z}\right|<\epsilon \quad(z \in K, n>N) .
$$


Der Beweis besteht aus einer ,quantitativen Version“ der Überlegungen, die zu (4) geführt haben. Für beliebige $z \in G$ gilt nach (3) die Abschätzung

$$
\left|f_{n}(z)-\sqrt{z}\right|=|\sqrt{z}| \cdot\left|\frac{1+\omega^{n}}{1-\omega^{n}}-1\right|=|\sqrt{z}| \cdot\left|\frac{2 \omega^{n}}{1-\omega^{n}}\right| .
$$

Da $K$ kompakt ist, gibt es ein $c>0$ mit $|\sqrt{z}| \leq c$ für alle $z \in K$ und wegen $|\omega(z)|<1$ auf $G \supset K$ auch ein $d<1$ mit $|\omega(z)| \leq d$ für alle $z \in K$. Hieraus folgt

$$
\left|\frac{2 \omega^{n}}{1-\omega^{n}}\right| \leq \frac{2\left|\omega^{n}\right|}{1-\left|\omega^{n}\right|} \leq \frac{2 d^{n}}{1-d} \quad(z \in K, n \geq 1) .
$$

$\mathrm{Zu}$ vorgegebenem $\epsilon>0$ gibt es nun ein $N$ mit $c \cdot 2 d^{n} /(1-d)<\epsilon$ für alle $n>N$, so dass (5) und (6) zusammen genommen die verlangte Abschätzung

$$
\left|f_{n}(z)-\sqrt{z}\right| \leq c \frac{2 d^{n}}{1-d}<\epsilon \quad(z \in K, n>N)
$$

liefern.

Bemerkung. Die Nullstellen der Polynomfolge $\left(p_{n}\right)_{n \geq 1}$ (wir haben sie oben bestimmt!) müssen auf der negativen reellen Achse dicht liegen. Andernfalls könnte nämlich das gemeinsame Holomorphiegebiet $G \operatorname{der} f_{n}$ bzw. der Grenzfunktion $\sqrt{ }$ vergrössert werden. Dies ist bekanntlich für $\sqrt{ }$ nicht möglich.

\title{
Literatur
}

[1] Fischer, W.; Lieb, I.: Funktionentheorie. 3., berichtigte Aufl., Vieweg 1983.

\author{
Werner Hertzog \\ Bauerngasse 6 \\ CH-4242 Laufen, Schweiz \\ e-mail: werner.hertzog@publica.ch
}

